\title{
CONTINUED FRACTION SOLUTIONS OF THE GENERAL RICCATI DIFFERENTIAL EQUATION
}

\author{
HOMER G. ELLIS
}

The ideas presented here were developed in response to an observation made by H. S. Wall, whose memory as teacher and friend I cherish. In a class meeting of his course on continued fractions (in $1959-60$, I think it was) he showed us how he had generated a continued fraction for a solution of the Riccati differential equation $y^{\prime}(x)=x^{2}+y^{2}(x)$. As an example for his undergraduate class in differential equations he had computed $y(x)$ as a ratio $u(x) / v(x)$ of power series satisfying $u^{\prime}(x)=x^{2} v(x), v^{\prime}(x)=-u(x), u(0)=0, v(0)=$ 1. He had then applied repeated long division and obtained

$$
y(x)=\frac{x^{3}}{3}-\frac{x^{4}}{7}-\frac{x^{4}}{11}-\frac{x^{4}}{15}-\ldots
$$

Recalling for us the similar continued fraction for $\tan x$, which satisfies the Riccati equation $y^{\prime}(x)=1+y^{2}(x)$, he proposed the problem of generalizing these expansions. I took up the challenge and after some experimentation arrived at the following iterative scheme: if $z$ is a complex number, and each of $p_{n}, q_{n}, r_{n}, a_{n}$, and $b_{n}$ is a complex-valued function on the real interval $I$, then the recurrence relation $y_{n}=$ $a_{n}{ }^{2} /\left(a_{n}-b_{n} z-y_{n+1} z^{2}\right)$ converts the Riccati equation $y_{n}{ }^{\prime}=p_{n}+$ $q_{n} y_{n} z+r_{n} y_{n}{ }^{2} z_{n}{ }^{2}$ to the Riccati equation $y_{n+1}^{\prime}=p_{n+1}+q_{n+1} y_{n+1} z+$ $r_{n+1} y_{n+1}^{2} z^{2}$ if

$$
\begin{aligned}
a_{n}{ }^{\prime} & =p_{n}, b_{n}{ }^{\prime}=q_{n} a_{n} \\
p_{n+1} & =p_{n}\left(b_{n} / a_{n}\right)^{2}-q_{n} b_{n}+r_{n} a_{n}^{2} \\
q_{n+1} & =2 p_{n}\left(b_{n} / a_{n}{ }^{2}\right)-q_{n} \\
r_{n+1} & =p_{n} / a_{n}^{2}
\end{aligned}
$$

Starting from $y_{0}=y, p_{0}=p, q_{0}=q, r_{0}=r$, this scheme produces, with minimal restrictions on $p$, $q$, and $r$, formal $J$-fraction solutions $y$ of the general Riccati equation

$$
y^{\prime}=p+q y z+r y^{2} z^{2}
$$

namely

$$
y=\frac{\alpha_{0}}{1-\beta_{0} z}-\frac{\alpha_{1} z^{2}}{1-\beta_{1} z}-\frac{\alpha_{2} z^{2}}{1-\beta_{2} z}-\ldots
$$

AMS (MOS) Subject Classifications (1970) Primary 34A45, Secondary 40A15. 
where $\alpha_{0}=a_{0}, \alpha_{n+1}=a_{n+1} / a_{n}$, and $\beta_{n}=b_{n} / a_{n}$. (There is an arbitrariness here in that at each step constants of integration for $a_{n}$ and $b_{n}$ appear.) As an example take $p(x)=x^{k}, q(x)=0, r(x)=x^{\ell}$, with $k+1>0$ and $k+\ell+2 \geqq 0$, and choose $a_{n}(0)=b_{n}(0)=0$. Then

$$
y(x)=\frac{x^{k+1}}{k+1}-\frac{x^{k+\ell+2} z^{2}}{2 k+\ell+3}-\frac{x^{k+\ell+2} z^{2}}{3 k+2 \ell+5}-\ldots
$$

This fraction converges for each value of $x$ to a function of $z$ that is meromorphic and is analytic at 0 . It reduces to Wall's example as well as to the analogous fraction for the tangent function.

Unable at the time to prove anything general about convergence of the $J$-fraction to an actual solution, I took another tack and considered formal power series solutions: if $y=c_{0}+c_{1} z+c_{2} z^{2}+\cdots$, then the Riccati equation (2) is a formal identity in $z$ if and only if

$$
\begin{aligned}
& c_{0}{ }^{\prime}=p, c_{1}{ }^{\prime}=q c_{0}, \text { and } \\
& c_{i+2}^{\prime}=q c_{i+1}+r\left(c_{0} c_{i}+c_{1} c_{i-1}+\cdots+c_{i} c_{0}\right) .
\end{aligned}
$$

Here again there is an arbitrariness of integration constants. For the choices of these consistent with $c_{n}\left(0^{+}\right)=0$ I determined usable conditions on $p, q$, and $r$ barely sufficient to cause the series to converge on an interval $(0, b)$ to an actual solution [1]. I have tried to make these conditions also imply convergence of the $J$-fraction, without success. The expectation that they should do so is based upon the intimate connection between the two kinds of formal solutions, as set forth in the following theorem.

THEOREM 1. If on the real interval I the complex-valued-function sequences $\left\{a_{n}\right\},\left\{b_{n}\right\}$, and $\left\{c_{n}\right\}$ are so related that

$$
\frac{\alpha_{0}}{1-\beta_{0} z}-\frac{\alpha_{1} z^{2}}{1-\beta_{1} z}-\frac{\alpha_{2} z^{2}}{1-\beta_{2} z}-\cdots
$$

is the formal J-fraction expansion of the series $c_{0}+c_{1} z+c_{2} z^{2}+\cdots$, where $\alpha_{0}=a_{0}, \alpha_{n+1}=a_{n+1} / a_{n}$, and $\beta_{n}=b_{n} / a_{n}$, then the $c_{n}$ satisfy the relations (5) on I if and only if the $a_{n}$ and the $b_{n}$ satisfy on I the relations (1) with $p_{0}=p, q_{0}=q$, and $r_{0}=r$.

The meaning of the $J$-fraction's being the formal expansion of the series is explained in [2, Chap. XI]. To prove the theorem it is easiest to observe that if

$$
\begin{aligned}
c_{0, n}+c_{1, n} z+c_{2, n} z^{2}+\cdots= & \\
& \frac{a_{n}{ }^{2}}{a_{n}-b_{n} z-\left(c_{0, n+1}+c_{1, n+1} z+c_{2, n+1} z^{2}+\cdots\right) z^{2}}
\end{aligned}
$$


is a formal identity in $z$, then (i) $c_{0, n}=a_{n}$, (ii) $c_{1, n}=b_{n}$, and (iii) the relations (5) hold with every letter additionally subscripted by $n$ if and only if $a_{n}{ }^{\prime}=p_{n}, b_{n}{ }^{\prime}=q_{n} a_{n}$, and (5) hold with $n+1$ as the additional subscript. Induction, starting from $c_{i, 0}=c_{i}, p_{0}=p, q_{0}=q, r_{0}=r$, then finishes the job.

The question whether the formal $J$-fraction solution, if convergent, represents a rigorous solution of the Riccati equation can be answered in part by application of 'I'heorem 1 and a theorem of Van Vleck [3, p. 15, and 2, p. 208] to establish the following conclusion.

THEOREM 2. If $M \geqq 0$, and at each point of I the formal J-fraction solution (3) converges uniformly for $|z| \leqq M$, then its limit satisfies the Riccati equation (2) for each $z$ such that $|z|<M$.

The reasoning is this: according to Van Vleck's theorem the power series expansion of the $J$-fraction has radius of convergence $M$ or more and sums to the $J$-fraction's limit; inside its circle of convergence it converges absolutely, with the consequence that it satisfies (2) exactly there, and not just formally.

The fraction in (4) converges as noted and therefore represents a rigorous solution of $y^{\prime}(x)=x^{k}+x^{\ell} y^{2}(x) z^{2}$. An extreme example in which convergence is not assured results from choosing $p(x)=K_{0}$, $q(x)=K_{1} \mid x, r(x)=K_{2} / x^{2}$. In this case the formal $J$-fraction solution for which $a_{n}\left(0^{+}\right)=b_{n}\left(0^{+}\right)=0$ is periodic:

$$
y(x)=\frac{K_{0} x}{1-K_{1} z}-\frac{K_{2} K_{0} z^{2}}{1-K_{1} z}-\frac{K_{2} K_{0} z^{2}}{1-K_{1} z}-\cdots .
$$

Unless $4 K_{2} K_{0} z^{2}\left(1-K_{1} z\right)^{-2}>1$, in which case it fails to converge, the fraction converges to $t x$, where $t$ satisfies

$$
t=K_{0}+K_{1} t z+K_{2} t^{2} z^{2}
$$

Study of this example suggested the convergence criterion in [1]. A different reflection upon it suggests the possibility of another formal solution of (2). Specifically, if $t^{\prime}$ is the other solution of (6), then $t^{\prime} x$ also is a solution of the Riccati equation; it has the obvious expansion

$$
t^{\prime} x=\frac{1-K_{1} z}{K_{2} z^{2}} x-t x=\frac{1-K_{1} z}{K_{2} z^{2}} x-\frac{K_{0} x}{1-K_{1} z}-\frac{K_{2} K_{0} z^{2}}{1-K_{1} z-\ldots}
$$

Thus the question arises: for the formal $J$-fraction solution (3) of the general Riccati equation does there exist a companion solution analogous to (7)? The answer is affirmative. If

$$
Y=\left(A-B z+Y_{0} z^{2}\right) / A^{2} z^{2},
$$




$$
\begin{aligned}
& \text { then } Y^{\prime}=p+q Y z+r Y^{2} z^{2} \text { if } Y_{0}^{\prime}=p_{0}+q_{0} Y_{0} z+r_{0} Y_{0}^{2} z^{2}, A^{\prime}=-r \text {, } \\
& B^{\prime}=-q A \text {, and } \\
& \quad p_{0}=p A^{2}-q B+r(B / A)^{2}, q_{0}=q-2 r\left(B / A^{2}\right), r_{0}=r / A^{2} .
\end{aligned}
$$

The previous algorithm is now applied to the equation for $Y_{0}$ to generate the $J$-fractional part of the expansion. Analogues of Theorems 1 and 2 can be established; the formal series solution that enters in is a Laurent series: if $y=d_{-2} z^{-2}+d_{-1} z^{-1}+d_{0}+d_{1} z+\cdots$, then the Riccati equation (2) is a formal identity in $z$ if and only if

$$
\begin{aligned}
& d_{-2}^{\prime}=r d_{-2}^{2}, d_{-1}^{\prime}=q d_{-2}+r\left(d_{-2} d_{-1}+d_{-1} d_{-2}\right), \\
& d_{0}^{\prime}=p+q d_{-1}+r\left(d_{-2} d_{0}+d_{-1} d_{-1}+d_{0} d_{-2}\right),
\end{aligned}
$$

and

$$
d_{n+3}^{\prime}=q d_{n+2}+r\left(d_{-2} d_{n+3}+d_{-1} d_{n+2}+\cdots+d_{n+3} d_{-2}\right) .
$$

By way of illustration, the companion to Wall's solution of $y^{\prime}(x)=$ $x^{2}+y^{2}(x)$ has the expansion

$$
Y(x)=-\frac{1}{x}+\frac{x^{3}}{5}-\frac{x^{4}}{9}-\frac{x^{4}}{13}-\frac{x^{4}}{17}-\cdots
$$

This is, not unexpectedly, the ratio $U(x) / V(x)$, where $U^{\prime}(x)=x^{2} V(x)$, $V^{\prime}(x)=-U(x), U(0)=1$, and $V(0)=0$.

\section{REFERENCES}

1. H. G. Ellis, Solvability of ordinary differential equations near singular points: an analytic case, Canad. J. Math. 19 (1967), 1303-1313.

2. H. S. Wall, Analytic Theory of Continued Fractions, Van Nostrand, Princeton, 1948.

3. E. V. Van Vleck, On the convergence of the continued fraction of Gauss and other continued fractions, Ann. of Math. (2) 3 (1901), 1-18.

4. I. V. Cygankov, Solution of Riccati equations by continued fractions, Solutions of a special Riccati equation by continued fractions, Perm. Gos. Univ. Učen. Zap. Mat. 17 (1960), no. 2, 99-107, 109-113. (See Math. Reviews 26 (1963), \#1520, \#1521, p. 293.)

5. E. P. Merkes and W. T. Scott, Continued fraction solutions of the Riccati equation, J. Math. Anal. Appl. 4 (1962), 309-327.

University of Colorado, Boulder, Colorado 80302 